\title{
Los retos académicos en época de pandemia
}

En este número de la Revista Colombiana de Neumología tenemos el gusto de presentar varios trabajos originales que tienen que ver con la utilización y la aplicación de pruebas de función pulmonar, como el de la espirometría para la evaluación de volúmenes y capacidades en sujetos exfumadores de más de 15 empresas del sector industrial de la ciudad de Cúcuta, Colombia (1). La investigación incluyó profesionales, técnicos, académicos y empleados de oficios varios, y fue un estudio transversal, descriptivo y observacional con 848 sujetos de ambos géneros, mayores de 16 años, exfumadores de más de un año de abandono. En ella se concluyó que el consumo del tabaco reduce los parámetros de las capacidades y los volúmenes pulmonares en personas, independientemente de las características antropométricas y que el abandono del hábito tabáquico puede prevenir las alteraciones en la función pulmonar (1). Todas las iniciativas que incluyan estudios alrededor del uso y/o la cesación del tabaco son bienvenidas, pues los neumólogos somos una pieza fundamental en estos proyectos. La Asociación tiene un Comité antitabaco muy activo con el cual se pueden articular en el futuro proyectos similares.

Se incluye también el artículo de la caminata de 6 minutos en población sana en una ciudad de nuestro país (2), el cual es un estudio descriptivo transversal que incluye 100 sujetos hombres y mujeres sanos entre los 18-80 años, en una institución de salud de la ciudad de Barranquilla, a quienes se les aplicó la caminata de seis minutos; otro artículo original tiene que ver con las exacerbaciones del asma en una clínica de cuarto nivel en Colombia (3); y, por último, encontramos los resultados de la primera clínica de anticoagulación perteneciente al sector de salud pública en Bogotá, que forma parte de la Red Distrital de Salud, la cual está adscrita a la Subred integrada de servicios de salud Centro Oriente, de la Secretaría Distrital de Salud de Bogotá (4). Además, en este número se publican varios reportes de caso muy interesantes. 
A pesar de los retos que nos ha impuesto la pandemia y la crisis de nuestro país, y del viraje de las prioridades hacia la atención de pacientes severamente comprometidos por el flagelo de la infección por el SARS-CoV-2, es muy meritorio el interés por desarrollar trabajos de investigación y reportes de casos difíciles en nuestros grupos académicos.

Asimismo, en este número tenemos la presentación oficial de un nuevo comité de nuestra Asociación, el

LILIANA FERnÁNDEZ-TRUJILLO

Editora en jefe

Revista Colombiana de Neumología
“Comité de la Mujer". La Dra. Alejandra Cañas, coordinadora del comité, y la Dra. Claudia Díaz presentan el nacimiento de esta iniciativa en un editorial adjunto.

Para finalizar, nos unimos a todas las familias de nuestros asociados que han tenido que sufrir en este momento crítico de la pandemia la pérdida de un ser querido, y presentamos el obituario de nuestro compañero y amigo Dr. Ernesto Agustín Santiago.

\section{Referencias}

1. Pérez-Lugo LM, Lobelo-Angulo JP, Varela-Prieto L, QuijanoDel Gordo CI, Santiago-Henríquez E. Distancia recorrida en la prueba de caminata de seis minutos en población adulta sana en una institución de salud de la ciudad de Barranquilla. Rev Colomb Neumol. 2021;32(2):20-26.

2. Moreno-Vega ML, Santiago-Barrios JP, Santiago E, VarelaPrieto L, Mora LA, Salcedo S. Exacerbación de asma en una clínica de IV nivel en Barranquilla: características clínicas y sociodemográficas. Rev Colomb Neumol. 2021;32(2):27-36.

3. Pereira-Rodríguez JE, Peñaranda-Flórez DG, Pereira-Rodríguez P, Pereira-Rodríguez R, Quintero-Gómez JC, Mauricio-Reynoso RA. Determinación por espirometría de volúmenes y capacidades pulmonares en sujetos exfumadores. Rev Colomb Neumol. 2021;32(2):10-19.

4. O Sáenz, F Sanabria, AM Rubio, F González, N Gamba. Clínica de anticoagulación de la Subred Centro Oriente, en Bogotá. Rev Colomb Neumol. 2021;32(2):37-45. 